\title{
Contribution à la systématique des Stenasellinae d'Afrique (Crustacés, Asellotes)
}

\author{
Par Guy Magniez ${ }^{1}$ )
}

Avec planches 3\%(1)-40(4)

Etudiant depuis 1960 la biologie des espèces françaises de Stenasellus, j'ai été amené à m'intéresser aux formes extra-européennes qui furent groupées dans ce genre et dont certaines particularités morphologiques se sont montrées pleines d'intérêt. C'est grâce à l'obligeance de Monsieur P. L. G. Benoit, Chef de la section des Invertébrés au Musée Royal de l'Afrique centrale à Tervuren (Belgique) et de Monsieur J. Forest, Sous-directeur au Museum National d'Histoire Naturelle de Paris que j'ai pu avoir communication du matériel provenant d'une part du bassin du Congo et d'autre part de l'Afrique occidentale. Je les en remercie bien vivement.

C'est à A. Viré que revint le mérite d'avoir découvert au gouffre de Padirac (Lot France) en 1896 ce genre d'Asellote dépigmenté et aveugle qui fut signalé et décrit très sommairement par A. Dollfus en 1897/98 sous le nom de Stenasellus; l'espèce-type étant dénommée virei. La nécessité de séparer cette nouvelle forme des Asellus épigés et hypogés justifiait parfaitement la création de cette coupure générique nouvelle. H. J. Hansen (1904) montra que le genre Stenasellus, possédant des caractères morphologiques très particuliers (notamment la fusion partielle entre tête et premier segment thoracique, le développement important des deux premiers pléonites et la constitution des mandibules), méritait même de constituer à lui seul une sous-famille spéciale parmi les Asellides. La justesse de ses vues est due à la grande connaissance qu'il avait de l'ensemble du groupe des Asellotes et en particulier des formes marines alors que la simple comparaison entre elles des seules formes dulçaquicoles mène à une impasse. A. Vandel (1938/47), étudiant les formules chromosomiques des Isopodes mit en évidence la totale discordance qui existe entre les diverses espèces

1) Laboratoire de Biologie Animale et Générale, Faculté des Sciences, 2 Bd. Gabriel, Dijon, France. 
d'Asellus et de Proasellus pour lesquelles le nombre haploïde est de 7 ou 8 grands chromosomes et Stenasellus virei pour lequel ce nombre est d'environ 27 minuscules éléments punctiformes.

E. G. Racovitza, qui disposa d'un abondant matériel à la suite des explorations méthodiques des grottes des Pyrénées faites en collaboration avec L. Fage et R. Jeannel, pût fournir une diagnose de l'espècetype St. virei. En 1924, 4 espèces de Stenasellus étaient ainsi décrites; la découverte d'une cinquième espèce d'Italie était annoncée et Racovitza pouvait alors donner une définition détaillée du genre.

A la suite de E. Dudich (1924), A. Arcangeli en 1938, dans une analyse critique détaillée des descriptions et publications antérieures montra que toutes les espèces de Stenasellus présentaient de telles différences structurales avec le genre Asellus qu'elles devaient constituer une famille distincte d'Asellotes; il donna la diagnose détaillée de la famille des Stenasellidae, qui comprenait le genre unique Stenasellus. Cette diagnose reprenait pour l'essentiel les critères définis par Racovitza en 1924 pour le genre lui-même. Il faut noter qu'Arcangeli s'appuyait à cette époque sur les descriptions des 8 formes alors connues, toutes européennes. Ce ne fut que quelques mois plus tard que parût la description du premier «Stenasellus» africain (St. chappuisi Remy 1938), qui aurait obligé Arcangeli à revenir quelque peu sur sa diagnose s'il avait pu alors en avoir connaissance.

De 1925 à 1962, 13 formes nouvelles d'Eurasie furent décrites, qui toutes répondaient remarquablement aux caractères génériques définis en 1924 (diagnose reprise par F. Frade en 1945). Ce qui nous donne la liste suivante :

Pour la Péninsule Ibérique et le Sud-Ouest de la France :

St. virei Dollfus 1897,

St. breuili Racovitza 1924,

St. buchneri Stammer 1936,

St. nobrei Braga 1942,

St. buili Remy 1949,

St. galhanoae Braga 1962.

Pour la Toscane et la Corse :

St. racovitzai Razzauti 1925.

Pour la Péninsule Balkanique (Yougoslavie, Hongrie, Bulgarie) :

St. gjorgjevici Racovitza 1924,

St. hungaricus Mehely 1924 ,

St. hungaricus robustus Mehely 1927,

St. bureschi Racovitza 1927, 
St. lakatnicensis Buresch 1927/62,

St. skopljensis Karaman 1935/36,

St. skopljensis croaticus Karaman 1954,

St. skopljensis meridionalis Karaman 1954,

St. hungaricus thermalis Mestrov 1960 ;

et pour le Turkestan :

St. asiaticus Birstein et Starostin 1949.

Dans le même temps, 6 formes africaines furent découvertes. Si nous faisons abstraction du Stenasellus guineensis Braga 1947 dont le mâle est encore inconnu et les affinités incertaines, ce sont :

Afrique occidentale :

St. chappuisi Remy 1938,

St. africanus Monod 1945.

Afrique equatoriale (bassin du Congo) :

St. leleupi Chappuis 1951,

St. congolensis Chappuis 1951,

St. dartevellei Chappuis 1952.

Toutes ces formes africaines, sauf africanus, présentent des variations structurales très importantes par rapport au type, sans aucune mesure avec celles qui affectent les espèces européennes entre elles. Ces variations sont incompatibles avec la diagnose générique proposée par Racovitza, si bien qu'il convient à l'heure actuelle de démembrer le grand genre Stenasellus selon les entités supra-spécifiques cohérentes qui s'y font jour.

Les formes $d u$ bassin du Congo méritent d'être réunies dans un genre spécial très homogène.

L'espèce chappuisi de Côte d'Ivoire et de Guinée portugaise qui paraît morphologiquement très isolée doit représenter à elle seule un autre genre particulier.

La famille des Stenasellidae, ramenée au rang de sous-famille par Vandel en 1964, ce que préconisait déjà prudemment Hansen en 1904, doit être définie de manière plus générale pour que les formes africaines répondent mieux à sa diagnose. Elle comportera alors les genres :

Stenasellus Dollfus s.str., qui comprend toutes les formes eurasiatiques ainsi que St.africanus Monod de Côte d'Ivoire. Cette espèce présente d'ailleurs une constitution tellement archaïque des pléopodes copulateurs du mâle (fig. 1A) que nous devons en faire mention séparément. Tout porte à croire que nous devrions aussi inclure dans le genre Stenasellus ainsi épuré le St.guineensis de Guinée portugaise, bien que les mâles fassent défaut. 
Metastenasellus nov.gen. pour les 3 espèces d'Afrique équatoriale dont les appareils copulateurs mâles ont évolué simultanément dans une voie commune.

Parastenasellus nov.gen. pour St.chappuisi Remy de Côte-d'Ivoire et de Guinée portugaise ; forme totalement différente de toutes les espèces tant africaines qu'eurasiatiques, avec des caractères qui évoquent ceux de certains Parasellidés.

Johannella Monod 1924, pour l'espèce algérienne Johannella purpurea Monod, qui initialement rapprochée des Mancasellus se révèle beaucoup plus voisine des Stenasellus que de tous les Asellides, plus évolués, et mérite de figurer à ce titre au sein de la sous-famille des Stenasellinae.

\section{Diagnose de la sous-famille des Stenasellinae}

Corps:

Céphalon plus large que long. Somite des maxillipèdes totalement incorporé à la capsule céphalique. Région génale normalement développée. Articulation céphalo-péréionale plus ou moins ankylosée médiotergalement. Clypeus plus large que long. Péréionite 1 présentant une forte concavité rostrale (fig. $2 \mathrm{~A}$ ).

Pléonites 1 et 2 toujours bien développés, de longueur variable (fig. 2I), mais toujours presqu'aussi larges que le péréionite 7 ; ils ne forment jamais un pédoncule rétréci au pléotelson (fig. 2B-C-E). Pléotelson toujours plus long que large (fig. 2 B-C-E-F ; $3 \mathrm{~A}-\mathrm{B}$ ).

\section{Appendices:}

Dernier article de l'antennule complètement fusionné avec l'avantdernier.

Rudiment d'exopodite à l'article 3 de la hampe de l'antenne.

Axe longitudinal du corps mandibulaire formant un angle presque droit avec l'axe longitudinal de l'apophyse dentaire apicale. Palpe triarticulé.

Lobe interne de la maxillule avec 5 tiges distales typiquement, dont certaines parfois très réduites.

Lobe interne de la maxille aussi large que les 2 autres portant une seule rangée de tiges tergales toutes semblables.

Maxillipède sans épipodite, avec un oostégite normal chez la femelle ovigère.

Péréiopodes semblables dans les deux sexes. Coxopodite du péréiopode 1 souvent relativement mobile chez les femelles ovigères. Dactyles 2 à 7 avec 1 ou 2 épines sternales. 
Pléopodes 1 s : Sympodites jamais totdlement coalescents entre eux. Rame toujours plus longue que le sympodite, avec une armature marginale de tiges plus ou moins développées (fig. $2 \mathrm{~J}-\mathrm{K}-\mathrm{L}$ ).

Pléopodes $2 \widehat{\sigma}$ : Exopodite biarticulé toujours plus petit que l'endopodite. Celui-ci typiquement biarticulé avec article distal conformé en pièce copulatrice (muni d'un canal ou d'une gouttière) (fig. $1 \mathrm{~A}-\mathrm{B}-\mathrm{D}-$ E-I-J).

Pléopodes $2 q$ : Subtriangulaires et petits, généralement libres entre eux ou à peine soudés proximalement (fig. $2 \mathrm{M}-\mathrm{N}$ ).

Pléopodes 3, 4, 5 : Exopodites 3 operculiformes, exopodites 4 et 5 plus longs que les endopodites correspondants, toujours chitinisés, parfois styliformes. Endopodites des trois paires charnus et respiratoires.

Pléopodes 5 toujours biramés.

Uropodes : Les deux rames de longueur comparable (fig. $2 \mathrm{~F} ; 3 \mathrm{~B}$ ).

Cette diagnose reprend pour l'essentiel le contenu de la définition générique de Stenasellus que nous devons à Racovitza et d'où est issue la diagnose de la famille des Stenasellidae donnée par Arcangeli. Cependant elle abandonne à la diagnose du genre Stenasellus s.str. les caractères trop restrictifs qu'elle tenait uniquement des formes européennes, c'est-à-dire surtout ceux liés à la conformation des pléopodes sexuels.

\section{Diagnose du genre Stenasellus s. str.}

Corps allongé, peu ou pas élargi au niveau des segments médians du péréion, yeux absents, pigmentation tégumentaire nulle, un pigment rose diffus dans les tissus.

Tergites des pléonites 1 et 2 bien développés, subquadrangulaires, de longueur environ moitié et de largeur 0,85 à 0,9 fois celles du péréionite 7 (fig. $3 \mathrm{~B}$ ).

Maxillipède normal.

Dactyles 2 à 7 avec 1 ou 2 épines sternales selon les espèces.

Sympodites des pléopodes 1 ô portant typiquement un rétinacle (fig. $2 \mathrm{~J}$ ).

Pléopodes 2 o : Endopodite biarticulé. Article proximal à section subcirculaire, grêle et long, uni à l'article distal par une articulation genouillée. Article distal toujours plus volumineux que le premier, de forme fusoïde variable, résultant de la transformation d'une lamelle chitineuse repliée médialement et dont les marges latérales se sont rapprochées (fig. 2D) et parfois légèrement soudées, sauf dans la région proximale où elles divergent. Les deux articles souvent de longueur subégale. Les articulations sympodite-premier article et 
premier article-second article sont très mobiles et permettent des mouvements de grande amplitude (fig. $1 \mathrm{~A}$ et I ; $3 \mathrm{C}$ et suivantes).

Pléopodes 2 q toujours libres (fig. $2 \mathrm{M}$ ).

Pléopodes 3,4,5: Les exopodites 4 et 5 sont typiquement des stylets chitineux, parfois des lames élargies, toujours plus longs que les endopodites correspondants. Endopodites charnus, respiratoires, de forme ovoïde simple, parfois bilobés.

Uropodes généralement aussi longs que le pléotelson.

Les pléopodes 1 du mâle recouvrent totalement les pléopodes 2 en position de repos.

Le genre Stenasellus privé des espèces congolaises et de $P$. chappuisi renferme encore toutes les formes eurasiatiques (17) plus les St.africanus Monod et guineensis Braga. Il reste done un ensemble important, mais très homogène cette fois, en ce qui concerne les grands traits de

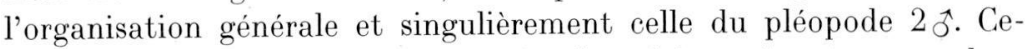
pendant, nous avons réuni côte-à-côte les schémas de cet organe dans les différentes espèces afin de montrer que des variations dans sa constitution de détail permettent de grouper certaines espèces entreelles :

Io-Article proximal de l'endopodite long et grêle :

a) Article distal isodiamétrique, à région distale large et armée de dents :

Stenasellus breuili Racovitza (fig. 3C),

Stenasellus galhanoae Braga (fig. $3 \mathrm{~F}$ ).

b) Article distal fusoïde à terminaison aiguë :

Stenasellus virei Dollfus (fig. 1 I),

Stenasellus racovitzai Razzauti (fig. $3 \mathrm{G}$ ),

Stenasellus buchneri Stammer (fig. $3 \mathrm{H}$ ),

Stenasellus nobrei Braga (fig. 3E),

Stenasellus buili Remy (fig. 3 I),

Stenasellus asiaticus Birstein \& Starostin (fig. 3D).

A l'intérieur de ce groupe, certaines espèces semblent très voisines ; c'est le cas de buchneri et nobrei.

$\mathrm{II}^{\circ}$-Article proximal de l'endopodite relativement court et trapu :

a) Article distal fusoïde volumineux et contourné, terminé en pointe :

Stenasellus skopljensis Karaman et ses deux sous-espèces, Stenasellus skopljensis meridionalis Karaman,

Stenasellus skopljensis croaticus Karaman (fig. 4E).

b) Article distal presque isodiamétrique à extrémité très obtuse avec un vaste orifice terminal : 
Stenasellus hungaricus Mehely (fi. 4F),

Stenasellus hungaricus robustus Mehely,

Stenasellus hungaricus thermalis Mestrov,

Stenasellus gjorgjevici Racovitza (fig. 4A),

Stenasellus bureschi Racovitza (fig. 4C),

Stenasellus lakatnicensis Buresch (fig.4D).

Certaines de ces formes seraient également très proches l'une de l'autre.

Nous ne pouvons pas rapprocher St.guineensis de l'un ou de l'autre groupe, faute de mâles, mais une remarque s'impose à propos du St.africanus qui ne figure pas non plus dans la tableau ci-dessus. La constitution de son organe copulateur montre l'aspect le plus primitif, le plus imparfait fonctionnellement parlant qu'on puisse imaginer. L'endopodite est une simple lame presque ovale, pratiquement plane, dont la marge externe porte un petit volet de même forme et plan lui-aussi (fig. 1A). Ces deux plans (fig. $4 \mathrm{G}$ ) forment entre eux un dièdre aigu. Ils préfigurent très imparfaitement l'entonnoir réalisé chez St.virei (section transversale 2D). Si nous ajoutons que pour la forme africaine les pléonites 1 et 2 montrent un développement en longueur plus important en valeur relative que pour les autres Stenasellus, que la lame interne de la maxillule porte 5 tiges de forte taille (fig. 4I), cette espèce bénéficie donc d'un ensemble exceptionnel de caractères primitifs dont il paraît essentiel de souligner l'existence.

\section{Diagnose du genre Metastenasellus ńov. gen.}

Corps allongé à bords très parallèles (fig. 2A-B-C). Yeux absents, pigmentation tégumentaire nulle, un pigment rose diffus dans les tissus.

Tergites des pléonites 1 et 2 très développés, subquadrangulaires, chacun de longueur égale aux $2 / 3$ environ, et de largeur égale à $8 / 9-9 / 10$ de celles du péréionite 7 (fig. $2 \mathrm{~B}-\mathrm{C}$ ).

Maxillipède normal.

Dactyles 2 à 7 avec une seule épine sternale.

Sympodite des pléopodes $1 \widehat{\sigma}$ sans rétinacle. Sympodite subcarré et rame subrectangulaire de même largeur que le sympodite (fig. $2 \mathrm{~K}$ ).

Pléopode 2્̧ : Sympodite subpentagonal, exopodite petit, endopodite secondairement uniarticulé, très volumineux, fusiforme, massif, parcouru par un canal hélicoïdal ouvert médio-proximalement et distalement. Endopodite pratiquement ankylosé sur le sympodite (fig. $1 \mathrm{~B}-\mathrm{D}-\mathrm{E}$ ). 
Pléopodes 29 plus ou moins soudés entre eux sur leur quart proximal (fig. $2 \mathrm{~N}$ ).

Pléopodes 3, 4, 5: Exopodites indurés, biarticulés, plus larges et plus longs que leur endopodite respectif. Endopodites ovoüdes, charnus respiratoires.

Uropodes à peine plus courts que le pléotelson (fig. 2C).

Les pléopodes 1 du mâle ne recouvrent pas entièrement les pléopodes 2 dont les stylets débordent largement en direction caudale au repos.

Habitat : Afrique centrale ; bassin hydrographique du Congo (grottes de la région de Thysville et sources de La Nouvelle Anvers). (Leleup 1949 ; Ghenne 1950.)

Espèces: Metastenasellus leleupi Chappuis 1951,

Metastenasellus congolensis Chappuis 1951,

Metastenasellus dartevellei Chappuis 1952.

Matériel: Provenant des collections du Musée Royal de l'Afrique Centrale à Tervuren.

Le caractère principal qui permet de distinguer Metastenasellus des autres Stenasellinae résulte de la conformation inhabituelle du pléopode copulateur (fig. 1 B). Nous pouvons, après Chappuis (1951), écrire que l'endopodite est uniarticulé. Fonctionnellement, il est fort peu mobile sur son sympodite et se présente comme un stylet rigide de taille importante, alors que dans les genres voisins, cet endopodite est constitué de deux articles bien distincts, de longueur souvent subégale et réunis par une articulation genouillée extrêmement mobile. Au repos, le stylet copulateur dépasse donc largement vers l'arrière la rame du pléopode 1 . Il repose contre la face sternale de l'opercule correspondant dort il atteint la suture médiane. Le pléopode 1 ne peut donc le protéger totalement comme cela se produit pour Stenasellus.

Chappuis en décrivant $M$.leleupi fut obligé d'admettre à priori que les deux articles composant primitivement cet endopodite étaient coalescents de façon à former un article unique (fig. 1D). Il supposa à cette occasion que la région proximale renflée du stylet représentait au moins en partie l'article proximal. Remarquons la structure très particulière de cette rame, parcourue de bout en bout par un canal hélicoïdal ouvert à ses deux extrémités (fig. 1 D-G). L'ouverture proximale est très proche de l'insertion (fig. $1 \mathrm{D}$ ), mais plus médiale, infundibuliforme à bords lisses, tandis que la distale a ses bords garnis de dents chitineuses, qui donnent à l'extrémité de l'endopodite l'aspect d'une «tête de serpent» (fig. $1 \mathrm{G}$ ). Chez M.leleupi adulte, la structure ne laisse plus deviner par quel processus s'est constitué un tel organe. Par contre, chez $M$.congolensis, des lignes de suture restent très nettement 
visibles (fig. $1 \mathrm{~B}-\mathrm{C}-\mathrm{F}$ ) : Le stylet s'est formé par torsion en hélice de l'article autour de son axe longitudinal, avec soudure des deux bords en contact, sauf dans la région proximale où subsiste un orifice et dans la région distale effilée, armée de dents, où la spire se resserre notablement. L'hélice étant dextre au pléopode gauche et senestre au pléopode droit, l'ensemble respecte la symétrie bilatérale de l'animal. La région tout à fait proximale de l'endopodite (au niveau de l'insertion) ne participe pas à la torsion. Elle semble partiellement séparée de la masse de l'endopodite et Chappuis y voit le premier article régressé et ankylosé de la rame. Pour M.dartevellei, la structure hélicoïdale est bien soulignée par une ligne de suture très visible, mais l'endopodite paraît tout à fait uniarticulé, avec un simple renforcement basal, si bien que Chappuis (1952) revient sur son interprétation de 1951 (pour M.congolensis) dans laquelle il faisait de cet anneau basal l'article proximal de l'endopodite (fig. $1 \mathrm{E}-\mathrm{H}$ ).

Après un nouvel examen des échantillons du Musée de Tervuren, il semble que l'endopodite du pléopode 2 ô s'est constitué selon un processus d'enroulement en hélice très particulier, mais identique pour les trois espèces congolaises (fig. $1 \mathrm{C}$ ). Sa structure est donc très voisine d'une espèce à l'autre et celles-ci sont indiscutablement apparentées. Il est probable que ce type de formation résulte d'une évolution poussée d'un endopodite biarticulé lamellaire par deux processus essentiels :

$1^{\circ}$-Réduction considérable de l'article proximal, ankylose de ses articulations, disparition de ses limites propres et naturellement de ses fonctions lors de l'accouplement.

$2^{\circ}$-Torsion en hélice (3 spires environ) de l'article distal rainuré longitudinalement sur sa face médiale, avec soudure des bords de cette gouttière, sauf proximalement, ce qui ménage une entrée au canal formé, et distalement, au niveau de l'armature copulatrice du stylet.

Fonctionellement, cette pièce copulatrice est bien plus parfaite que dans le genre Stenasellus pour lequel elle demeure généralement un entonnoir asymétrique très imparfait. Ici, cet endopodite canaliculé constitue une véritable seringue à injection si l'on sait que les extrémités des papilles génitales, qui sont très longues, viennent au contact des orifices proximaux des endopodites par simple rotation des pléopodes 2 autour de leur axe d'insertion transverse. La perfection fonctionnelle de l'appareil copulateur de Metastenasellus est à rapprocher de celle de l'endopodite du pléopode 2 ô des Asellus, qui résulte de processus morphogénétiques très différents. C'est pour M.leleupi que l'évolution paraît la plus poussée, puisque toute trace de suture hélicoïdale constitutive a disparu, de même que disparaît 
chez les Aselles évolués la trace de l'invagination sternale de la vésicule interne de la pièce copulatrice. Il est donc possible de constituer un groupe de trois espèces présentant une unité structurale certaine, tout en différant suffisamment entre elles pour qu'aucune confusion ne soit possible. Les structures perfectionnées de leurs pléopodes $2 \widehat{\jmath}$, différant tant de ceux des Stenasellinae ouest-africains que de ceux des formes eurasiatiques permettent de définir avec précision le genre congolais Metastenasellus nov.gen. dont le préfixe voudrait souligner le degré supérieur de perfectionnement de l'appareil copulateur mâle. Si ce perfectionnement va de pair avec la possession des pléonites 1 et 2 les plus archaïques du groupe, il est une nouvelle preuve de l'indépendance des vitesses et des directions évolutives de deux caractères morphologiques distincts.

Diagnose du genre Parastenasellus nov.gen.

Corps relativement trapu (allongement 10/3), nettement élargi au niveau des péréionites médians (largeur maximale au niveau du $4^{\circ}$ chez la femelle et du $6^{\circ}$ chez le mâle) (fig. $2 \mathrm{E}-\mathrm{F}$ ). Yeux absents, pigmentation tégumentaire nulle.

Tergites des pléonites 1 et 2 peu développés en longueur (fig. $2 \mathrm{H}$ ), en forme de croissants à concavité caudale et recouverts partiellement par la partie postérieure de celui du péréionite 7 . Leur longueur est de $1 / 4$ environ, leur largeur de $5 / 6$ environ de celles du péréionite 7 (fig. $2 \mathrm{~F}$ ).

Maxillipède portant chez le mâle un endite charnu équivalent de l'oostégite de la femelle (fig. 2O-P).

Dactyles 2 à 7 avec une seule épine sternale.

Sympodites des pléopodes $1 \hat{\jmath}$ triangulaires très réduits, sans rétinacle et divergents. Rame parcheminée, réfléchie sur ses bords interne et distal, enveloppant le pléopode 2 et garnie seulement de fines soies marginales. L'appendice rappelle celui de certains Parasellides (fig. 2L-G).

Pléopodes 2 ○ै: Sympodite triangulaire avec une apophyse axiale terminale. Les deux rames sont portées par la marge interne. Endopodite biarticulé grêle genouillé ; l'article distal est fusoüde falciforme, avec une gouttière longitudinale, sa section est plus faible que celle de l'article proximal. L'appendice rappelle aussi celui de certains Parasellides (fig. $1 \mathrm{~J}-\mathrm{K}-\mathrm{L}$ ).

Pléopodes 2 + totalement indépendants.

Pléopodes 3, 4, 5 : Exopodites lamelleux subovalaires biarticulés. L'exopodite 3 déborde marginalement le pléotelson. Endopodites 3, 4 et 5 bilobés et charnus. 
Uropodes de longueur environ $2 / 3$ de celle du pléotelson (fig. $2 \mathrm{~F}$ ).

Habitat : Afrique occidentale : Côte d'Ivoire (Chappuis 1931); Guinée portugaise (Pimenta 1947 ; Gata 1948).

Matériel : Provenant des collections du Muséum National d'Histoire Naturelle, Paris.

L'Asellote découvert dans le puits du poste de Man (Côte d'Ivoire) par Chappuis en 1931, décrit par Remy en 1938, a été retrouvé en 1947 et 1948 et identifié par Braga (1950) dans le puits de l'Administration de Gabu (Guinée portugaise) à $1000 \mathrm{~km}$. à vol d'oiseau de la station originelle. C'est donc probablement une forme à vaste répartition, peuplant les nappes phréatiques au contact de la croûte latéritique et de sa roche-mère dans toute cette zone de l'Afrique Occidentale. Les microcristaux de produits latéritiques se retrouvent en abondance dans le contenu intestinal de l'animal.

Parastenasellus chappuisi Remy nov.gen. possède des caractères morphologiques qui l'éloignent de tous les autres Stenasellinae. Cette espèce isolée doit donc constituer un genre particulier dont nous venons de donner la diagnose. Il convient maintenant de faire ressortir les points suivants :

La très forte réduction de longueur des pléonites 1 et 2 , dont les plaques tergales prennent une forme en croissant à concavité caudale qui se retrouvera chez Johannella (fig. $2 \mathrm{~F}-3 \mathrm{~A}$ ). Médialement, les tergites de ces pléonites sont largement inclus sous celui du péréionite 7 (fig. 2 E).

Le caractère essentiel porte sur la constitution des pléopodes sexuels du mâle. Les pléopodes 1 avec leur sympodite atrophié, triangulaire, sans rétinacle et leur rame parcheminée, à marges médiale et distale réfléchies vers la face tergale, qui enveloppe et protège totalement l'organe copulateur correspondant sont d'un aspect inhabituel chez les Stenasellinae (fig. $2 \mathrm{~L}-\mathrm{G}$ ). Les pléopodes 2, eux aussi, par leur structure, évoquent ceux des Parasellidae (Janira sp., par exemple) (fig. $1 \mathrm{~J}-\mathrm{K}$ ). Le sympodite triangulaire se termine par une apophyse axiale obtuse et son bord médial possède une profonde cavité où s'insèrent les deux rames : endopodite copulateur géniculé à article proximal subcylindrique plus court que l'article distal qui, de forme fusoïde aiguë, présente une gouttière longitudinale à ouverture subterminale (fig. $1 \mathrm{~K}$ ) dont l'observation est difficile. Ces appendices évoquent ceux des Parasellides d'eau douce qui peuplent certaines grottes et sources de l'Afrique du Sud (Protojanira Barnard 1927 et Anneckella Chappuis et Delamare 1957) (fig. 1L-2 G).

Notons enfin la présence régulière aux maxillipèdes du mâle d'un endite charnu qui simule l'oostégite réduit de la femelle en période de 
repos génital (fig. 2O-P). Chez la femelle ovigère, l'oostégite devient très grand, mais reste glabre comme chez Stenasellus. Le matériel de Man contient de très nombreuses femelles à divers stades, mais seulement une dizaine de mâles. Parmi ceux-ci, deux présentent une allure intersexuée notable : Le premier possède un oostégite au péréiopode 1 gauche et pour le second, l'oostégite existe aux péréiopodes 1, 2, 3 gauches, 1 et 2 droits. Les papilles génitales sont normalement développées avec des amas de spermatozoïdes dans leur canal et les pléopodes 1 et 2 parfaitement constitués ; anomalie que nous n'avons jamais encore constatée chez les autres Stenasellinae. Les mâles sont anormalement rares, puisque les pêches répétées de l'espèce en Guinée portugaise n'ont jamais fourni que des femelles. Notons encore la très curieuse cohabitation de $P$. chappuisi avec un autre Stenasellinae dans ses deux stations connues (St.africanus Monod à Man, St.guineensis Braga à Gabu), fait unique dans le groupe.

\section{Diagnose du genre Johannella Monod}

Corps trapu (allongement 2,45/1), contour général du péréion fortement convexe. Largeur maximale au droit des péréionites 4 et 5 . Yeux absents, pigmentation tégumentaire nulle, un pigment rouge diffus dans les tissus.

Tergites des pléonites 1 et 2 en croissant à concavité caudale, de largeur $7 / 9$ de celle du péréionite 7 (fig. $3 \mathrm{~A}$ ).

Maxillipède normal.

Dactyles 2 à 7 avec une seule épine sternale.

Pléopodes 1 ơ inconnus.

Pléopodes 2 ơ inconnus.

Pléopodes 2 q soudés médialement sur leur tiers proximal.

Pléopodes 3, 4, 5: Exopodite 3 comme dans les autres genres, le reste inconnu.

Uropodes extrèmement courts.

Habitat : Algérie ; source sulfureuse chaude $\left(29^{\circ}\right)$ d'El Hamman ; (Gauthier 1924).

Johannella purpurea Monod (1924) a été décrite après la capture d'un seul individu femelle. Ses caractères morphologiques sont tels que c'est à juste titre que l'auteur y vit un genre nouveau d'Asellote. Sa constitution générale (développement important des pléonites 1 et 2 , fusion totale du somite des maxillipèdes avec la capsule céphalique, concavité rostrale du péréionite 1 , courbure de la mandibule à angle droit, présence d'une seule rangée de tiges tergales au lobe interne de la maxille, maxillipèdes apparemment sans épipodite, pléo- 
podes $2 q$ triangulaires et petits) (fig. $3 \mathrm{~A}$ ), permet d'y voir indiscutablement une forme voisine des Stenasellus. D'autres indices, tels que la pigmentation rouge diffuse dans les tissus sont en faveur de cette opinion, et enfin, il fût pèché dans une source chaude. Nous savons que les eaux thermales représentent un refuge par excellence des formes anciennes (dont Stenasellus hungaricus thermalis Mestrov 1960 nous fournit un autre exemple). Pourtant, le fait que le mâle de $J$.purpurea demeure inconnu nous interdit de préciser davantage la position du genre par rapport aux autres Stenasellinae et en particulier de l'assimiler à Parastenasellus, comme on pourrait penser le faire.

\section{Conclusion}

Les Asellotes archaïques, phréatiques ou cavernicoles, qui avaient été réunis dans le genre unique Stenasellus peuvent se répartir en trois groupes qui comptent respectivement 19, 3 et 1 formes et desquels il faut rapprocher Johannella purpurea Monod. Ces quatre genres diffèrent suffisamment entre eux pour qu'aucune confusion ne soit possible et leurs diagnoses respectives montrent de nombreux caractères différentiels. Le genre Stenasellus demeure africain et eurasiatique, groupant des espèces qui s'échelonnent de la Côte d'Ivoire au Turkestan, avec une grande richesse de formes périméditerranéennes. Le genre Metastenasellus se cantonne pour l'instant dans les régions congolaises et quoique située sur le même continent, son aire de répartition connue est aussi éloignée des stations de Stenasellus et Parastenasellus de l'Afrique occidentale que celles-ci le sont des stations européennes les plus méridionales de Stenasellus. La situation géographique du genre Johannella ne constitue en aucune façon une anomalie. La faune d'Asellotes de l'Afrique, bien qu'encore relativement pauvre en espèces connues, se montre très diversifiée, puisque outre les Stenasellinae, l'Afrique du Nord possède une faune d'Aselles épigés et hypogés du groupe coxalis typiquement méditerranéenne, et l'Afrique australe abrite dans ses eaux douces épigées et souterraines des formes spéciales de Parasellides (Carte $4 \mathrm{H}$ ). La pénétration de ces Parasellides essentiellement marins en eau douce a été signalée à maintes reprises (Dollfus 1889 ; Margaleff 1952 ; Maury 1927, entre autres) et le peuplement des eaux douces interstitielles par les Microparasellides est une autre illustration de ce phénomène de colonisation.

En ce qui concerne les rapports exacts de Stenasellus et genres voisins avec les autres Asellotes, toute précision demeure aléatoire. Hansen donna en 1904 une diagnose des familles Asellidae, Stenetriidae et Parasellidae. Les caractères distinctifs portaient presque exclusive- 
ment sur les pléopodes 1, 2 et 3 et dans la définition des Asellides nous trouvons: «pléopodes $2 \mathrm{du}$ mâle petits, situés sous les pléopodes 1 et non couplés à eux. Rames fixées sur la marge distale du sympodite. Endopodite non genouillé ; son article distal renflé contenant une grande cavité et son extrémité obtuse. Exopodite presque aussi long que l'endopodite, son article distal mobile lamelleux, avec des soies marginales...» Il est bien évident qu'une telle définition, pas plus que celle de Grüner (1965) ne peut s'appliquer aux Stenasellinae. Par ailleurs, Hansen notait que par des caractères tels que l'ankylose partielle entre tête et premier péréionite, l'important développement des deux premiers pléonites, Stenasellus diffère de tous les autres Asellotes pour lesquels les pléonites 1 et 2 réduits en longueur et en largeur figurent un pédoncule entre péréion et pléotelson. D'autres caractères sont propres aux Stenasellinae (courbure à $90^{\circ}$ de la mandibule, absence d'épipodite au maxillipède, mais présence d'un oostégite fonctionnel à cet appendice par exemple). Par contre la présence d'un rudiment d'exopodite à l'antenne et la structure genouillée de l'endopodite copulateur sont des caractères qu'ils possèdent en commun avec les Stenetriidae ou les Parasellidae. Stenasellus et les genres apparentés possèdent done des caractères morphologiques qui les rapprochent des Asellides, d'autres communs avec les Stenetrioidea et Paraselloidea et surtout des caractères propres d'importance fondamentale. C'était déjà l'opinion de Dudich (1924) et d'Arcangeli (1938). La situation des Stenasellinae au voisinage des Asellides doit donc être considérée comme une position d'attente provisoire, toujours susceptible de revision à la faveur de découvertes ultérieures.

\section{RÉSUMÉ}

De 1897 à 1962, de nombreuses espèces ont été décrites comme appartenant au genre Stenasellus Dollfus. Or certaines formes : celles de l'Afrique Centrale et une forme de l'Afrique occidentale présentent des caractères qui ne sont pas conformes à la diagnose générique formulée par Racovitza en 1924, ce qui oblige à créer pour elles les genres nouveaux Metastenasellus et Parastenasellus. Les Asellotes archaïques appartenant aux genres Stenasellus Dollfus, Johannella Monod, Metastenasellus nov.gen. et Parastenasellus nov.gen. sont donc groupés dans la sous-famille des Stenasellinae dont les rapports avec les divers groupes d'Asellotes actuels restent à préciser.

\section{ZUSAMMENFASSUNG}

In den Jahren 1897 bis 1962 wurden der Gattung Stenasellus Dollfus zahlreiche Spezies zugeordnet. Einige unter ihnen, nämlich die zentralafrikanischen und eine westafrikanische, weichen in gewissen Merkmalen dermaßen von der Gattungsanalyse Racovitzas (1924) ab, daß die Aufstellung neuer 
Gattungen erforderlich wird. Zwei neue Genera werden beschrieben: Metastenasellus und Parastenasellus. Die auf die Gattungen Stenasellus Dollfus, Johannella Monod, Metastenasellus nov.gen. und Parastenasellus nov.gen. sich verteilenden archaischen Aselloten bilden die Unterfamilie der Stenasellinae, deren Beziehungen zu den verschiedenen Gruppen der heutigen Aselloten noch zu präzisieren sind.

\section{B I B LI OGRAP H I E}

Arcangeli, A. (1938) - Stenasellus racovitzai Razzauti, Crostaceo Isopodo acquatico cavernicolo italiano. La famiglia Stenasellidae. Arch. Mus. Zool. Anat. Comp. Torino, 46, 17-50.

Barnard, K. H. (1927) - A study on the freshwater Isopodan and Amphipodan Crustacea of South-Africa. Trans. Roy. Soc. S.Afr., 14, 139-215.

- (1955) - A new Protojanira from Natal (Isopoda Asellota). Ann. Natal Mus., 13, 249-251.

Birstein, J. A. (1951) - Faune de l'URSS. Vil/5, Asellota. Moscou, 1-142.

Birstein, J. A., et Starostin, I. V. (1949) - Sur une forme nouvelle pour l'URSS de Stenasellus provenant du Turkmenistan (en russe). C. R. Acad. Sc. Moscou; N.S., 69, 691-694.

Braga, J. M. (1950) - Sur deux Stenasellus (Crust. Isop.) de la Guinée portugaise. Ann. Fac. Sc. Porto, 35, 50-56.

- (1962) - Sur la distribution géographique des Stenasellus de la Péninsule Ibérique et desçription d'une espèce nouvelle de ce genre. Ann. Fac. Sc. Porto, 44, 9-28.

Brian, A., et Dartevelle, E. (1949) - Contribution à l'étude des Isopodes marins et fluviatiles du Congo. Ann. Mus. Congo belge, Tersuren, C. Zool., (3), vol. 1 (2), 78-208.

Buresch, I., et Gueorguiev, V. (1962) - Contribution à la connaissance des espèces du genre Stenasellus (Crustacea Asellota) en Bulgarie avec description d'une nouvelle espèce. Bull. Inst. Zool. et Mus., 11, 189-206.

Chappuis, P. A. (1951) - Isopodes et Copépodes cavernicoles. Rev. Zool. Bot. Afr., 44, 4, 342-359.

- (1952) - Un nouveau Stenasellus du Congo belge. Res. Zool. Bot. Afr., 45 , $353-357$.

Chappuis, P. A., et Delamare-Deboutteville, Cí. (1957) - Un nouvel Asellide de l'Afrique du Sud. Notes biospél., 12, 29-36.

Delamare-Deboutteville, Cl. (1960) - Biologie des eaux souterraines ; littorales et continentales. Suppl. à Vie et Milieu, 9, 1-740.

Dollfus, A. (1889) - Description d'un Isopode fluviatile du genre Jaera provenant de l'île de Florès (Açores). Bull. Soc. zool. France, 14, 133-134.

- (1897) - Sur deux types nouveaux de Crustacés Isopodes appartenant à la faune souterraine des Cévennes. C.R.Acad. Sc. Paris, 125, 130-131.

Dudich, E. (1924) - Über Protelsonia hungarica Méhely. Zool. Anz., 60, $151-155$. 
Frade, F. (1945) - Crustaceos dulciaquicolas de Portugal. Bol. no 26 Com. Fisc. Aguas de Lisboa, 91-98.

Grindey, J. R. (1963) - A new Protojanira (Crustacea Isopoda) from a Cape pensinsula cave. Ann. Transwaal Mus., 24, 4, 271-274.

Grüner, H. E. (1965) - In: Dahl, F.: Die Tierwelt Deutschlands, 51, Krebstiere oder Crustacea, V/1, 1-149.

Hansen, H. J. (1904) - On the morphology and classification of the Asellotagroup of Crustaceans, with descriptions of the genus Stenetrium Hasw. and its species. Proc. Zool. Soc. London, 2, 302-331.

Husson, R. (1957) - A propos de la biologie du Crustacé troglobie aquatique Stenasellus virei Dollfus. Ann. Uniø. Saraviensis, Sc., 6, 74, 8259-8269.

Karaman, S. (1954) - Über die jugoslawischen Stenasellus-Arten. Fragm. Balcan. Mus. Macedon. Sc. Nat., 1, 7-20.

Leleup, N. (1956) - La faune cavernicole du Congo Belge et considérations sur les Coléoptères reliques d'Afrique intertropicale. Ann. Mus. R; Congo belge, Zool., 46, 1-165.

Lemercier, A. (1960) - La super-espèce Jaera nordmanni (Rathke) (Isopodes, Asellotes, Janiridae). Crustaceana, 1, 1, 9-27.

Magniez, G. (1963) - A propos d'une anomalie de certaines femelles de Stenasellus virei Dollfus (Crustacé Isopode Troglobie). Ann. Spéléol. Paris, 18, 4, 483-490.

MargalefF, R. (1952) - Une Jaera dans les eaux douces des Baléares, Jaera balearica nov.sp. (Isopoda Asellota). Hydrobiologia, 4, 209-213.

Mavry, A. (1927) - Une nouvelle station dulçaquicole de Jaera marina (Fabricius) (Crustacé Isopode). Bull. Soc. linn. Normandie, 7, 9, 103-105.

Mestrov, M. (1960) - Stenasellus hungaricus thermalis ssp.n. (Crustacea Isopoda), Fund einer vorglazialen Art in warmen Quellen bei Zagreb. Biol. Glasnik, 13, 345-350.

Monod, Тн. (1924) - Sur quelques Asellides nouveaux des eaux douces de l'Afrique du Nord. Bull. Soc. Hist. Nat. Afr. Nord, 15, 7, 327-336.

- (1932) - Sur un Asellus aberrant (A.remyi nov.spe.) du lac d'Ohrid (Albanie). Bull. Soc. Zool. France, 57, 3, 206-217.

- (1945) - Un nouveau Stenasellus ouest-africain. Bull. I.F.A.N., 7, 101-114.

Racovitza, E. G. (1924) - Diagnose des genres Asellus et Stenasellus et description de deux Stenasellus nouveaux. Bull. Soc. Sc. Cluj, 2, 2, 81-92.

- (1950) - Asellides - première série - : Stenasellus. Arch. Zool. exp. gén. Paris, 87, 1-94.

Remy, P. (1938) - Un Stenasellus en Afrique occidentale française : Stenasellus chappuisi n.sp. de la Côte d'Ivoire. Arch. Zool. exp. gén. Paris, $79,69-74$.

- (1948) - Sur quelques Crustacés cavernicoles d'Europe. Notes biospéol Paris, 3, 35-47.

- (1949) - Stenasellus buili n.sp. de la grotte de la Giraudasso à Soulatgé, Aude (Crustacé Isopode). Bull. Soc. linn. Lyon, 18, 7, 153-157. 
Stammer, H. J. (1936) - Eine neue Höhlenwasserassel aus Spanien, Stenasellus buchneri, und die Verbreitung der Gattung Stenasellus. Zool. Anz., 114, 137-141.

Vandel, A. (1938) - Les chromosomes des Asellotes. C.R. Acad. Sc. Paris, $206,621-623$.

- (1947) - Recherches sur la génétique et la sexualité des Isopodes terrestres-X-Etude des garnitures chromosomiques de quelques espèces d'Isopodes marins, dulçaquicoles et terrestres. Bull. biol. FranceBelgique, 81, 1/2 ,154-176.

- (1964) - Biospéologie - La biologie des animaux cavernicoles. GauthierVillars, Paris, 1-619.

\section{EXPLICATION DES PLANGHES 37(1)-40(4)}

\section{PLANCHE $37(\mathbf{1})$}

A - Stenasellus africanus : pléopode $2 \overbrace{}^{\star}$ gauche, face sternale. B - Metastenasellus congolensis : pl. $2 \sigma^{+}$droit, face tergale. $\mathrm{C}-$ M.congolensis : pl. $2 \sigma^{\hat{\sigma}}$ gauche, face tergale d'un jeune de $7,80 \mathrm{~mm}$., la torsion n'est pas encore achevée. D - M.leleupi : pl. $2 \hat{0}$, d'après Chappuis (1951). E-M.dartevellei : pl. 20ิ, d'après Chappuis (1952). F - M.congolensis : extrémité de l'endopodite de pl. $2 \hat{o}$ à fort grossissement. G-M.leleup $i$ : idem. H - M.dartevelle $i$ : idem, d'après Chappuis (1952). I - Stenasellus sirei : pl. $2 \sigma^{\star}$ gauche, face sternale. J - Parastenasellus chappuisi : pl. $2 \hat{\jmath}$ droit, face sternale. K - Extrémité de l'endopodite du précédent à fort grossissement. L - Anneckella ficki: pl. 2ð, d'après Chappuis et Delamare (1957).

\section{PLANCHE $38(2)$}

A ; B - M.congolensis, vue tergale. C - M.leleupi : face tergale du pléon. D - St.virei: section transverse au tiers distal du second article de l'endopodite du pl. $2 \hat{\sigma}$. E - P. chappuisi $i$ : face tergale du pléon. F - P. chappuisi $\widehat{\delta}$ : pléon artificiellement distendu pour mettre en évidence les tergites des pléonites 1 et 2. G-Anneckella ficki: pl. 2 + , d'aprés Chappuis et Delamare (1957). H - P. chappuisi : coupe parasagittale des tergites du péréionite 7 et des pléonites 1 et 2 . I - St.virei : même coupe, le péréionite 7 est vers le bas. J-St.virei : pl. 1 o $^{\star}$ droit, face sternale. K-M.leleupi: pl. 1 ô $^{\star}$ d'un adulte. L - P. chappuisi: pl. $1 \delta^{\uparrow}$ droit, face tergale. M - St. virei : pl. 2 + gauche, face sternale. N - M.congolensis : pl. 2 + , face sternale. $\mathrm{O}-P$. chappuisi ô de

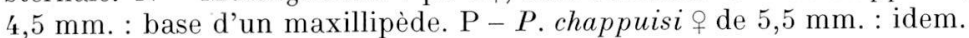

\section{PLANCHE $39(3)$}

A - Johannella purpurea : face tergale du pléon, d'après Monod (1924). B - St.virei : idem, d'après Racovitza (1950). G - St.breuili : pléopode 20ิ, d'après Racovitza (1924). D - St.asiaticus : idem, d'après Birstein (1951). E - St.nobrei : idem, d'après Braga (1942). F - St.galhanoae : idem, d'après Braga (1962). G-St.racovitzai : idem, d'après Arcangeli (1938). H - St.buchneri: idem, d'après Braga (1962). I - St.buili: idem, d'après Remy (1949).

\section{PLANCHE $40(4)$}

A ; B - St.gjorgjevici : pl. 2ồ et extrémité de son endopodite, d'après Racovitza (1924). C - St.bureschi : pl. 20ิ, d'après Buresch et Gueorguiev (1962). D - St.lakatnicensis : idem. E - St.skopljensis croaticus : idem, 
d'après Karaman (1954). F - St.hungaricus : idem, d'après Remy (1948). G-St.africanus : Endopodite copulateur; la lamelle accessoire de l'article distal rabattue artificiellement dans le plan de celui-ci. H - Carte schématique de la répartition des Stenasellinae et de quelques autres Asellotes d'Afrique. Les cercles, carrés, croix, signalent les stations. (St.sp. = différentes espèces du genre Stenasellus s.str. ; J.p. = Johannella purpurea ; P.c. $=$ Parastenasellus chappuisi $;$ St.g. $=$ Stenasellus guineensis $;$ St. a. $=$ Stenasellus africanus ; M.c., M.l., M.d. = Metastenasellus congolensis, leleupi, dartevellei; A.f. $=$ Anneckella ficki $;$ P.j. $=$ Protojanira sp.) I - St. africanus : extrémité de la lame interne de la maxillule.

Les échelles sont en microns pour les dessins originaux, en kilomètres pour la carte. 


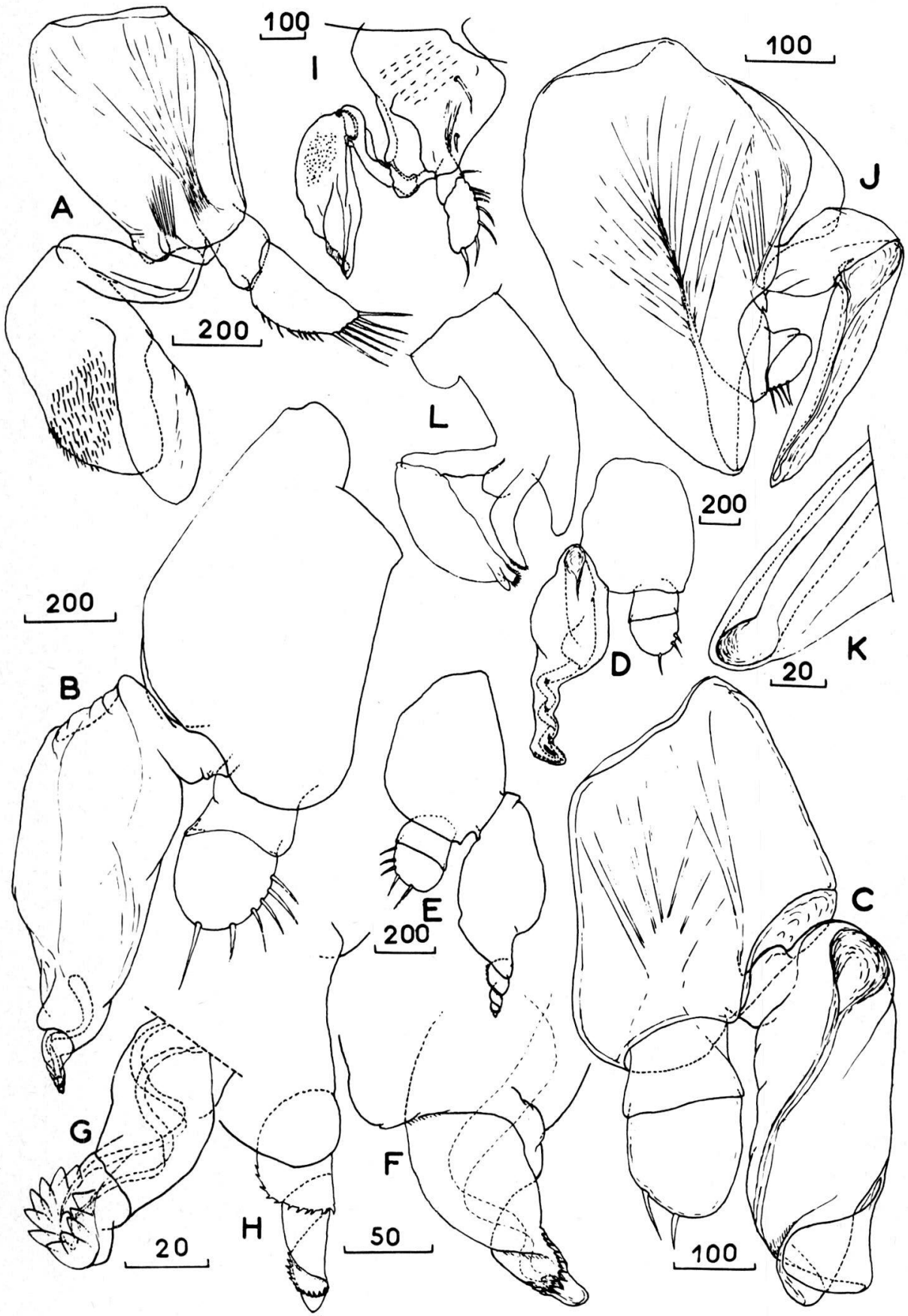




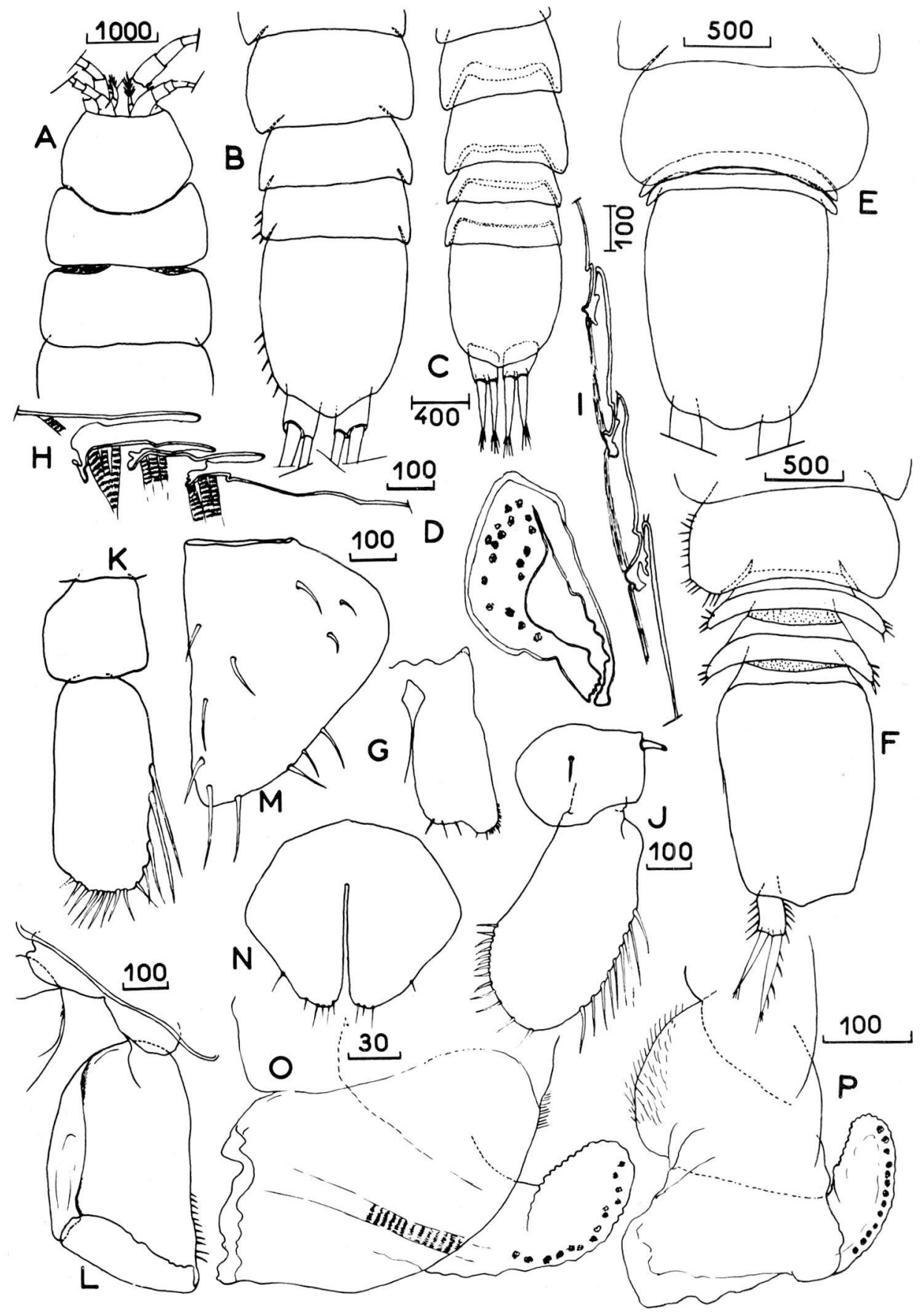




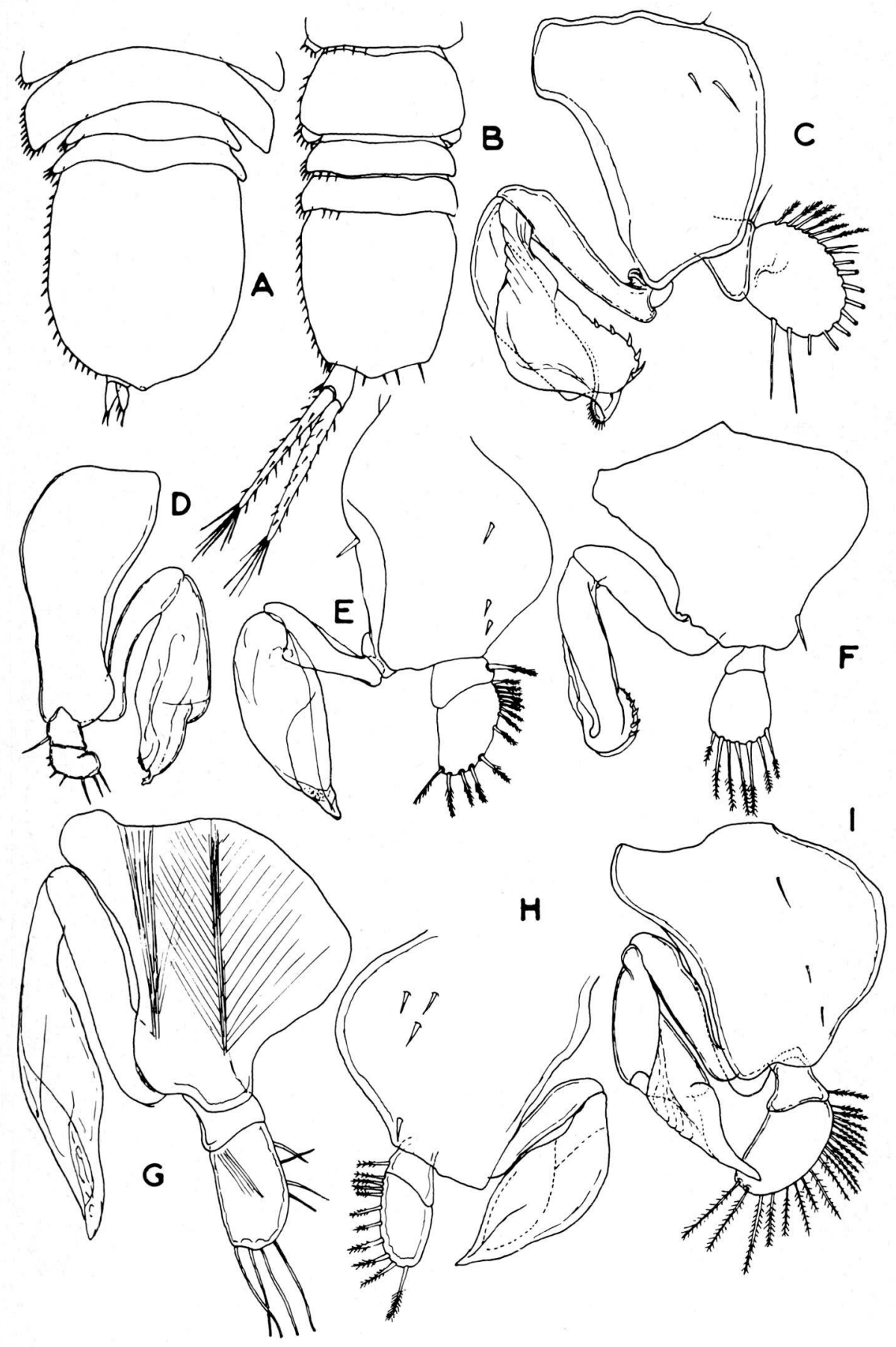




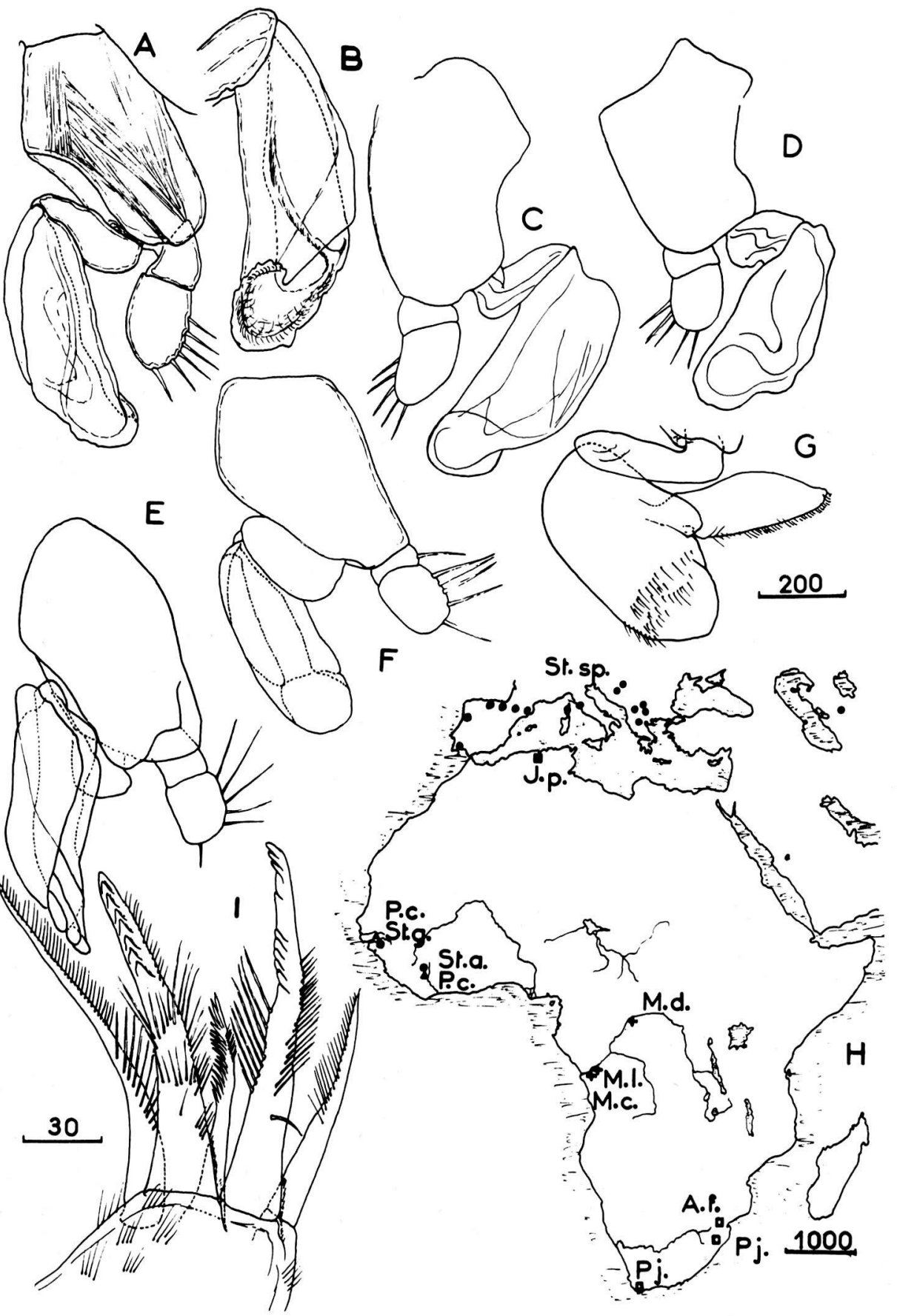

